\title{
Glucocorticoid programing of the mesopontine cholinergic
} system

\section{Sónia Borges ${ }^{1,2}$, Bárbara Coimbra ${ }^{1,2}$, Carina Soares-Cunha ${ }^{1,2}$, Ana P. Ventura-Silva ${ }^{1,2}$, Luisa Pinto ${ }^{1,2}$, Miguel M. Carvalho ${ }^{1,2}$, José-Miguel Pêgo ${ }^{1,2}$, Ana João Rodrigues ${ }^{1,2 *}$ and Nuno Sousa ${ }^{1,2}$ *}

${ }^{1}$ Life and Health Sciences Research Institute (ICVS), School of Health Sciences, University of Minho, Braga, Portugal

2 ICVS/3B's - PT Government Associate Laboratory, Braga/Guimarães, Portugal

\author{
Edited by: \\ Nikolaos P. Daskalakis, Icahn School \\ of Medicine at Mount Sinai, USA \\ Reviewed by: \\ Therese Jay, INSERM U894, France \\ Li-Tung Haung, Chang Gung \\ University College of Medicine, \\ Taiwan \\ *Correspondence: \\ Ana João Rodrigues and Nuno Sousa, \\ Life and Health Sciences Research \\ Institute (ICVS), School of Health \\ Sciences, University of Minho, \\ Campus de Gualtar, 4710-057 Braga, \\ Portugal \\ e-mail: ajrodrigues@ \\ ecsaude.uminho.pt; \\ njcsousa@ecsaude.uminho.pt
}

Stress perception, response, adaptation, and coping strategies are individually distinct, and the sequel of stress and/or glucocorticoids (GCs) is also distinct between subjects. In the last years, it has become clear that early life stress is a powerful modulator of neuroendocrine stress-responsive circuits, programing intrinsic susceptibility to stress, and potentiating the appearance of stress-related disorders such as depression, anxiety, and addiction. Herein we were interested in understanding how early life experiences reset the normal processing of negative stimuli, leading to emotional dysfunction. Animals prenatally exposed to GCs (in utero glucocorticoid exposure, iuGC) present hyperanxiety, increased fear behavior, and hyper-reactivity to negative stimuli. In parallel, we found a remarkable increase in the number of aversive $22 \mathrm{kHz}$ ultrasonic vocalizations in response to an aversive cue. Considering the suggested role of the mesopontine tegmentum cholinergic pathway, arising from the laterodorsal tegmental nucleus (LDT) and pedunculopontine tegmental nucleus (PPT), in the initiation of $22 \mathrm{kHz}$ vocalizations and hypothetically in the control of emotional arousal and tone, we decided to evaluate the condition of this circuit in iuGC animals. Notably, in a basal situation, iuGC animals present increased choline acetyltransferase (ChAT) expression in the LDT and PPT, but not in other cholinergic nuclei, namely in the nucleus basalis of Meynert. In addition, and in accordance with the amplified response to an adverse stimulus of iuGC animals, we found marked changes in the cholinergic activation pattern of LDT and PPT regions. Altogether, our results suggest a specific cholinergic pathway programing by prenatal GC, and hint that this may be of relevance in setting individual stress vulnerability threshold.

Keywords: glucocorticoids, stress, acetylcholine, anxiety, fear, pedunculopontine tegmental nucleus, laterodorsal tegmental nucleus, ultrasonic vocalizations

\section{INTRODUCTION}

Exposure to stressful events or synthetic glucocorticoids (GCs), such as dexamethasone, early in life, are a risk factors for the development of different neuropsychiatric disorders in adulthood, namely depression and anxiety (1). Such effects are partially mediated by de-regulation of the hypothalamic-pituitaryadrenal (HPA) axis, leading to altered GC secretion $(2,3)$, which can induce long-term molecular and functional changes in GCsensitive nuclei. Importantly, several recent studies showed that high levels of GCs have a deleterious effect in the developing brain, inducing prominent neurochemical, structural, and molecular changes in several brain regions (4-8) culminating in the development of anxious and depressive-like traits (9-13).

GC programing effects in emotional behavior are far from being completely understood at a mechanistic level, but it has been shown that GCs modulate on the long-term the activity of particular neural pathways. In this perspective it is interesting to refer that early life stress/GC exert a powerful effect in the developing dopaminergic neurons, especially at the mesolimbic level (7). For example, we have shown that animals prenatally exposed to GC present marked hypodopaminergia and D2 epigenetic/expression changes, responsible for their anhedonia and motivational deficits, since administration of a dopamine precursor, L-DOPA, fully reverted the molecular and behavioral impairments $(14,15)$. Obviously, this dopaminergic de-regulation may also occur indirectly through modulation of upstream neurotransmitter systems. One promising candidate is the ascending cholinergic pathway, comprising projections from acetylcholine (ACh)-rich nuclei within the pons, particularly the laterodorsal (LDT) and pedunculopontine tegmental nuclei (PPT) to the ventral tegmental area (VTA) (16-18). In support of this, it was already shown that stress/GC strongly elicit cholinergic activity of these regions (19), and that they, in turn, critically affect basal and phasic activity of VTA neurons (20-23). Additionally, GC can putatively bind to GC-responsive elements of cholinergic players and control their expression, namely choline acetyltransferase (ChAT) (24) and acetylcholine esterase (AChE) (25), two enzymes of the cholinergic pathway. Also relevant is to note that the ascending cholinergic system is implicated in the control of the stress response by modulating hypothalamic pituitary adrenal (HPA) axis function (26-28) and in mediating the anxiogenic effects of stress (29-31). 
Surprisingly, very few studies have focused on the impact of stress/GC exposure in the cholinergic system. Considering the lack of evidence, herein, we focused on the impact of prenatal exposure to GCs in the ascending cholinergic system, and the relevance of such changes in stress-related anxious and fear behaviors.

\section{MATERIALS AND METHODS \\ ANIMALS AND TREATMENTS}

All manipulations were conducted in accordance with local regulations on animal care and experimentation (European Union Directive 2010/63/EU). Pregnant Wistar Han rats were subcutaneously injected with the synthetic GC dexamethasone at $1 \mathrm{mg} \mathrm{kg}^{-1}$ (in utero glucocorticoid exposure, iuGC animals), or with vehicle (CONT; control animals), on days 18 and 19 of gestation.

Dexamethasone dosage was selected based on our previous studies showing that this regimen effectively impairs HPA axis activity in a long-term basis (12). From a clinical perspective, guidelines on prenatal corticotherapy $(32,33)$ recommend single course administration $(0.3-0.5 \mathrm{mg} / \mathrm{kg})$, however, multiple courses of GCs are often administered (34), despite the lack of evidence of increased therapeutic efficacy. Nevertheless, we must consider the difficulty in the transposition of human doses to rodents due to ADME species specificity.

At weaning day, male offspring were house-paired randomly, according with prenatal treatment, under standard laboratory conditions: artificial $12 \mathrm{~h}$ light/dark cycle (lights on from 08:00 a.m. to 08:00 p.m.); room temperature $22^{\circ} \mathrm{C}$; food and water were provided ad libitum. Animals derived from at least three different litters were used for all the experimental procedures.

\section{BEHAVIORAL TESTS}

All tests were performed during the day period, except the confined cage and fear-conditioning protocols that were conducted during the night period (08:30 p.m. to 03:00 a.m.). All behavioral equipment was cleaned between animals (ethanol 10\%) in order to remove any olfactory cues.

\section{OPEN FIELD}

The open field (OF) test was conducted in an arena $(43.2 \mathrm{~cm} \times 43.2 \mathrm{~cm})$ with transparent acrylic walls and white floor (Med Associates Inc., St. Albans, VT, USA). Rats were placed in the center of the arena and movement was monitored over a period of $10 \mathrm{~min}$ with the aid of two 16-beam infrared arrays. Total distance traveled was used as an indicator of locomotor activity.

\section{ELEVATED PLUS MAZE}

The elevated plus maze (EPM) test was carried out under bright white light. Animals were placed individually for $5 \mathrm{~min}$ in the center of a black polypropylene plus-shaped platform elevated $72.4 \mathrm{~cm}$ above the floor. The apparatus consisted of two open arms $(50.8 \mathrm{~cm} \times 10.2 \mathrm{~cm})$ and two closed arms $(50.8 \mathrm{~cm} \times 10.2 \mathrm{~cm} \times 40.6 \mathrm{~cm})$ (MedAssociates Inc., St. Albans, VT, USA). The number of entries into each arm and the time spent therein were recorded.

\section{LIGHT/DARK BOX TEST}

The light/dark box (L/D) test was performed inside the OF arena $(43.2 \mathrm{~cm} \times 43.2 \mathrm{~cm})($ MedAssociates Inc., St. Albans, VT, USA). A dark compartment was attached to one side with an opening facing the center of the arena. Animals were individually placed in the center of the illuminated part. The distance traveled and time spent in each compartment was recorded in a single trial of $10 \mathrm{~min}$.

\section{CONFINED CAGE TEST}

The confined cage test was performed in a non-restrictive Plexiglas cylinder (inner diameter $8.8 \mathrm{~cm}$, length $22.2 \mathrm{~cm}$ ), mounted on a Plexiglas platform and placed in a ventilated, sound-attenuated chamber (SR-LAB, San Diego Instruments, San Diego, CA, USA). A stainless steel grid was placed inside the cylinder, through which an electric current could be passed (shock chamber). A microphone and a video camera were placed inside the sound-attenuated chamber. The protocol was performed in two consecutive days, in which the animals were placed inside the shock chamber for $3 \mathrm{~min}$. The ultrasonic vocalizations (USVs) and the percentage of total freezing time were measured.

\section{FEAR-CONDITIONING PARADIGM}

The fear-conditioning test was performed in a non-restrictive Plexiglas cylinder (inner diameter $8.8 \mathrm{~cm}$, length $22.2 \mathrm{~cm}$ ), mounted on a Plexiglas platform and placed in a ventilated, sound-attenuated chamber (SR-LAB, San Diego Instruments, San Diego, CA, USA). The protocol was performed in three consecutive days (35). On the first day (habituation), each animal was placed in the shock chamber for $11 \mathrm{~min}$. On the second day (conditioning), each subject was positioned inside the shock chamber for 3 min (no light, no shock). Afterwards, animals were exposed to six lights/shock pairings $(0.4 \pm 0.1 \mathrm{~mA})$, with an inter-stimulus-interval (ISI) of $60 \mathrm{~s}$. The shock was given for $500 \mathrm{~ms}$, immediately after the cue light was turned off. On the following day (test day), after an initial phase of $3 \mathrm{~min}$ without light, animals were presented with a $20 \mathrm{~s}$ cue light for six times, but no shock was given (ISI of $60 \mathrm{~s}$ ). During all procedures, USVs and freezing behavior were recorded.

\section{USVs ANALYSIS}

An ultrasound microphone (CM16/CMPA, Avisoft Bioacoustics, Berlin, Germany) sensitive to frequencies of $10-200 \mathrm{kHz}$, placed $15 \mathrm{~cm}$ above the floor, was used in all experiments. The microphone was connected via an Avisoft UltrasoundGate 416H (Avisoft Bioacoustics) to a personal computer; USVs were recorded using the Avisoft-Recorder (version 5.1.04) with the following settings: sampling rate: 250,000; format: 16 bit. For acoustical analysis, recordings were transferred to Avisoft SASLab Pro (version 5.1.22, Avisoft Bioacoustics). This program was used in order to produce spectrograms of USVs by conducting a fast Fourier transformation (256 FFT-length, 100\% frame, Hamming window filter, 50\% time window overlap). These spectrograms had a frequency resolution $\sim 1.2 \mathrm{kHz}$ and a temporal resolution $\sim 0.4 \mathrm{~ms}$.

Twenty-two kilohertz call detection was provided by an automated threshold-based algorithm (threshold: $-40 \mathrm{~dB}$ ) and a hold time mechanism (hold time: $20 \mathrm{~ms}$ ). A lower-cut-off-frequency of $18 \mathrm{kHz}$ was used to reduce background noise. 
Calls were also inspected manually to ensure that, when necessary, USVs not detected automatically could be subsequently included in the automatic parameter analysis.

\section{IMMUNOHISTOCHEMISTRY}

For immunohistochemistry (IHC), 11 rats were sacrificed by pentobarbital (Eutasil, Sanofi) anesthesia and transcardially perfused with $0.9 \%$ saline followed by $4 \%$ paraformaldehyde $(\mathrm{pH} 7.4$ in $0.1 \mathrm{M}$ phosphate buffer). Brains were removed and post-fixed for $48 \mathrm{~h}$ in $4 \%$ paraformaldehyde and then rinsed and stored in $30 \%$ of sucrose until sectioning. Brains were sectioned coronally, at a thickness of $50 \mu \mathrm{m}$, on a vibratome (VT1000S, Leica, Germany) and stored in cryoprotectant solution at $-20^{\circ} \mathrm{C}$ until use.

Briefly, free-floating sections were pre-treated with $3 \% \mathrm{H}_{2} \mathrm{O}_{2}$ in PBS, thoroughly rinsed in PBS, blocked with $2.5 \%$ fetal bovine serum (FBS) in PBS-Triton $0.3 \%$ for $2 \mathrm{~h}$ at room temperature, and then incubated overnight at $4^{\circ} \mathrm{C}$ with primary antibody goat anti-ChAT (Millipore, MA, USA; 1:1000). Afterwards, sections were washed, incubated with the secondary biotinylated anti-goat (Vector Lab., USA; 1:200) for $1 \mathrm{~h}$, and processed with an avidin-biotin complex solution (ABC-Elite Vectastain reagent; Vector Lab, USA). Detection was done using $0.5 \mathrm{mg} / \mathrm{ml}$ $3,3^{\prime}$-diaminobenzidine. Sections were washed and mounted on glass slides, air-dried, counterstained with hematoxylin, and cover slipped with Entellan-New (Merck, Darmstadt, Germany).

\section{IMMUNOFLUORESCENCE}

Ninety minutes after completion of the fear-conditioning paradigm, 12 animals were deeply anesthetized with pentobarbital (Eutasil, Sanofi) and were transcardially perfused with $0.9 \%$ saline followed by $4 \%$ paraformaldehyde. Brains were removed and post-fixed in $4 \%$ paraformaldehyde. Coronal vibratome sections $(50 \mu \mathrm{m})$ were first incubated with rabbit anti-c-fos primary antibody (1:1000; Ab-5, Calbiochem, USA), followed by incubation with goat anti-ChAT primary antibody (1:1000; anti-ChAT, Millipore, MA, USA). Secondary fluorescent antibodies were: Dylight 488-conjugated donkey anti-rabbit IgG (1:500, BioLegend), and Alexa Fluor 568-conjugated donkey anti-goat IgG (1:500, Invitrogen), respectively. Finally, all sections were stained with $4^{\prime}, 6$-diamidino-2-phenylindole (DAPI; $1 \mathrm{mg} / \mathrm{ml}$ ). For each animal, c-fos-positive cells within the PPT and LDT were analyzed after double staining with cholinergic (ChAT) marker and cell counts were performed by confocal microscopy (Olympus FluoViewTMFV1000, Hamburg, Germany). Estimation of cell density was obtained by crossing cell number values with the corresponding areas, determined using an Olympus BX51 optical microscope and the StereoInvestigator software (Microbrightfield, VT, USA).

\section{STEREOLOGICAL PROCEDURES}

Eleven animals were perfused transcardially with $4 \%$ paraformaldehyde, under deep pentobarbital (Eutasil, Sanofi) anesthesia. Brains were included in glycolmethacrylate (Tecnovit 7100, Heraeus Kulzer, Wehrheim, Germany) and sectioned on a microtome as described in detail elsewhere (36). Every other $30 \mu \mathrm{m}$ thick coronal section was collected on a gelatinized slide, stained with Giemsa, mounted with Entellan-New (Merck, Darmstadt, Germany), and cover slipped. Stereological procedures were performed by a blind observer.

Laterodorsal tegmental and PPT regions were outlined according to the atlas of Paxinos and Watson (37) and based on noticeable cytoarchitectural differences, namely density of cells and size of the perikarya.

Volume and neuronal number estimations were performed using StereoInvestigator software (Microbrightfield, VT, USA) and a camera attached to a motorized microscope (Axioplan 2, Carl Zeiss, Germany). The Cavalieri's principle was used to assess volume. Briefly, every second section was used and the cross-sectional area was estimated by point counting (final magnification $112 \times$ ). We used a test point system in which the interpoint distance, at the tissue level, was $150 \mu \mathrm{m}$ for LDT and $200 \mu \mathrm{m}$ for PPT. The volume of the region of interest was calculated from the number of points within its boundaries and the distance between sampled sections.

Average cell numbers were estimated using the optical fractionator method (38). Briefly, a grid of virtual 3D-boxes (LDT: $30 \mu \mathrm{m} \times 30 \mu \mathrm{m} \times 20 \mu \mathrm{m}$; PPT: $40 \mu \mathrm{m} \times 40 \mu \mathrm{m} \times 20 \mu \mathrm{m}$ ) equally spaced (using the same grid spacing as for volume estimations) was superimposed on every second section of the lamina of interest and cells within boxes were counted. Coefficients of error (CE) were automatically computed, according to the formulas of Gundersen et al. (39) for cell numbers and Gundersen et al. (40) for volume estimations. Glial cells were not included in the estimations, and the discrimination between neuronal and glial cell body profiles was based on the criteria described by Ling et al. (41) and Peinado et al. (42).

\section{STATISTICAL ANALYSIS}

Statistical analysis was performed in GraphPad Prism 5.0 (GraphPad Software, Inc., La Jolla, CA, USA). Statistical analysis between two groups was made using Student's $t$-test or Mann-Whitney tests. Two-way analysis of variance (ANOVA) was used when appropriate. Bonferroni's post hoc multiple comparison test was used for group differences determination. Non-parametric analysis (Mann-Whitney test) was used when normality of data was not assumed. Results are presented as mean \pm SEM. Statistical significance was accepted for $p \leq 0.05$.

\section{RESULTS \\ IN UTERO GLUCOCORTICOID EXPOSURE IMPAIRS EMOTIONAL BEHAVIOR}

Animals were exposed to a battery of behavioral tests that consisted of paradigms studying spontaneous exploratory behavior $(\mathrm{OF})$, tasks of innate anxiety (EPM, L/D test, confined cage), and reactivity to adverse stimulus (version of fear-conditioning paradigm). Since USVs can give information on the emotional status of the animal, we decided to further complement the behavioral characterization by measuring USVs in these different paradigms.

In the OF, iuGC animals presented a decrease in the number of ambulatory counts (Figure 1A, $t=2.197, p=0.037$ ) and total distance traveled (Figure 1B, $t=3.002, p=0.006$ ) when compared with control animals. In addition, iuGC animals exhibited a decrease in the percentage of time spent in the center of the arena (Figure 1C, $t=2.416, p=0.023$ ). 


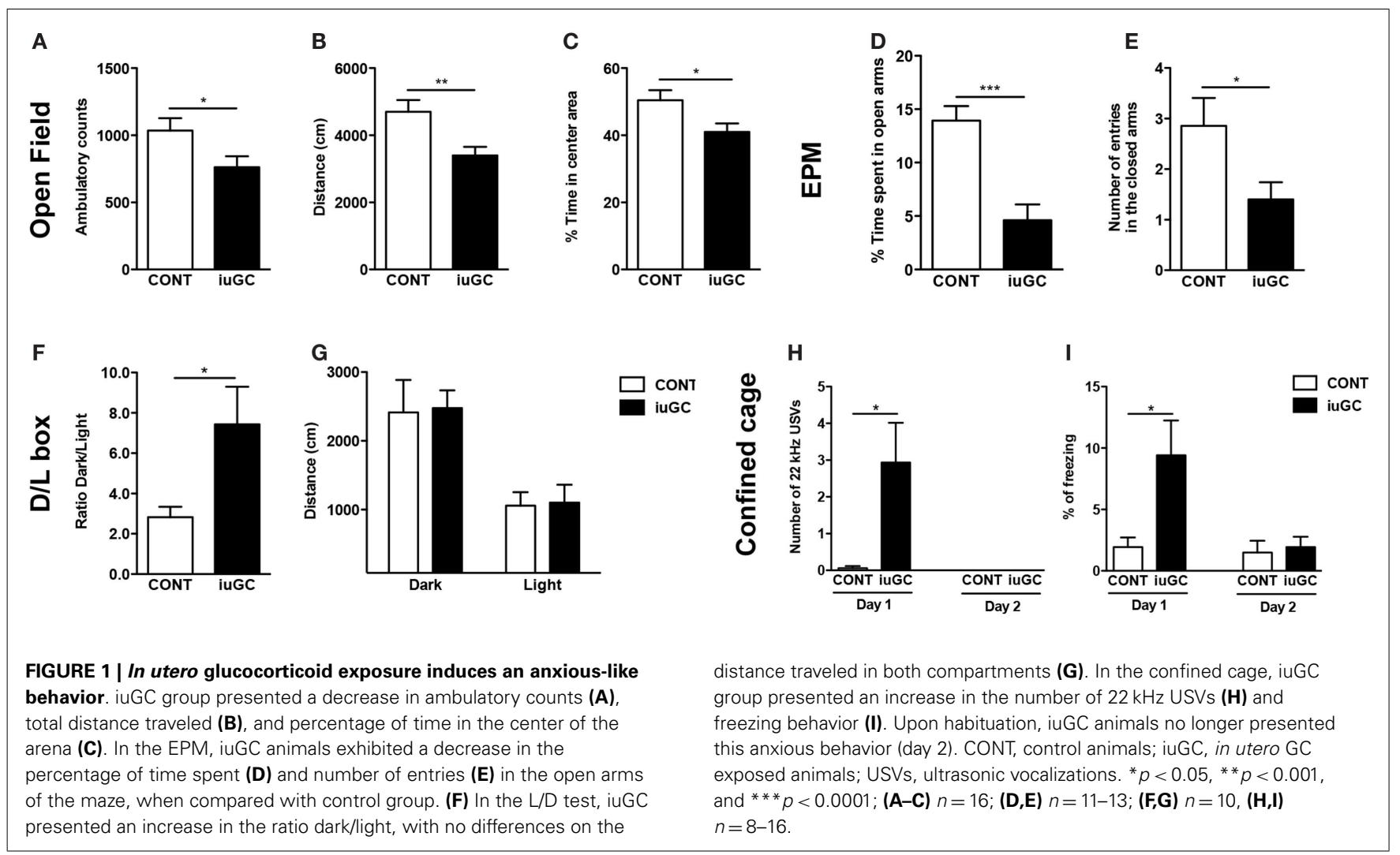

In the EPM, iuGC animals spent significantly less time in the open arms (Figure 1D, $t=2.947, p=0.009$ ) and presented a reduction in the number of open arms entries (Figure 1E, $t=2.375, p=0.031$ ), when compared with control animals. No differences were found in the time and entries in the closed arms (data not shown; time: $t=0.479, p=0.637$; entries: $t=0.872$, $p=0.392$ ).

In the $\mathrm{L} / \mathrm{D}$ test, iuGC animals presented an increase in the ratio dark/light (Figure 1F, $t=2.765, p=0.014$ ) and no differences in distance traveled in both compartments (Figure 1G, dark: $t=0.117, p=0.909$; light: $t=0.137, p=0.893$ ).

In the confined cage, while control animals rarely emitted aversive $22 \mathrm{kHz}$ USVs, iuGC animals vocalized throughout the exposure (Figure 1H; $U=81, p=0.016$ ). Similarly, iuGC group presented increased freezing behavior (Figure 1I; $t=2.846$, $p=0.013$ ). Upon habituation to the cage (2 days of exposure), iuGC animals no longer presented this anxious-like response.

To investigate reactivity to an adverse stimulus, we performed a variation of the classical fear-conditioning paradigm. Animals were conditioned to a cue (light) predicting painful electric shocks. After cage habituation, animals were given six pairs of cue-shock. No emission of $22 \mathrm{kHz}$ USVs was observed in the baseline phase (0-3 min), but upon light/shock pairings, as expected, both groups significantly emitted more negative USVs. iuGC animals emitted negative USVs to a greater extent than control animals (Figure 2A, $t=1.562, p=0.130)$ and also presented an increase in freezing behavior time (Figure 2B, $t=2.355, p=0.034$ ). After this conditioning session, in the next day, animals were exposed to the cue,

but no shock was given. In the initial period, both groups emitted more $22 \mathrm{kHz}$ USVs than the day before $\left(F_{1,58}=4.10, p=0.047\right)$; cue exposure elicited more negative USVs in both groups, but again, iuGC animals were over-reactive (Figure 2C, $t=2.804$, $p=0.011)$. Analysis of freezing behavior further confirmed the phenotype of iuGC group (Figure 2D, $t=2.087, p=0.049$ ). Plotting the number of negative USVs along time further confirms that iuGC animals were over-reactive to the cue predicting the adverse stimulus, since iuGC group emitted more context-induced $22 \mathrm{kHz}$ vocalizations than control group, especially in the first $60 \mathrm{~s}$ (Figure 2E, $F_{1,131}=104.42, p<0.0001$ ). Upon the first cue exposure (ON period), both groups increased the number of USVs with no major differences between them. However, upon second cue exposure, iuGC animals emitted more negative USVs than control group and remained over-reactive throughout. The pattern of $22 \mathrm{kHz}$ USVs emission in both groups is interesting since immediately after the light is turned off, both groups emit more negative vocalizations, indicative of the consolidated association of the cue with the electric shock.

\section{iuGC EXPOSURE INDUCES PROMINENT CHOLINERGIC ALTERATIONS}

Pharmacological studies suggest that the ascending cholinergic tegmental system is responsible for the initiation and production of negative vocalizations in rodents (43-45). In addition, cholinergic signaling is highly responsive to stress/GC (46) and is important for the manifestation of aversive behaviors (47). Considering these findings, we decided to further explore the impact of iuGC exposure in the cholinergic circuitry. To do so, 

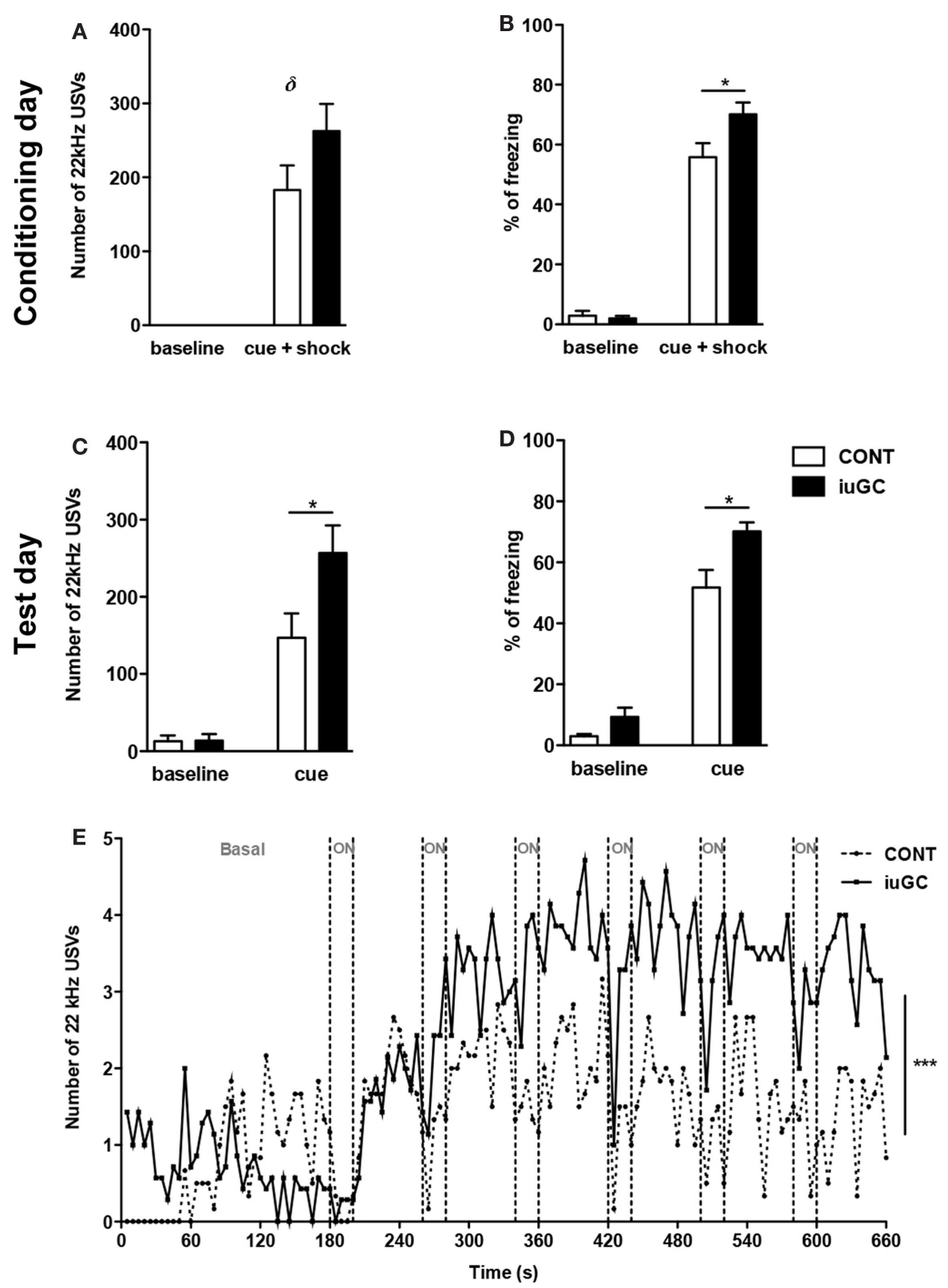

FIGURE 2 | Prenatal exposure to GC leads to amplified response to adverse stimulus. (A) Number of $22 \mathrm{kHz}$ USVs on the conditioning day (cue + shock) of the fear-conditioning paradigm. iuGC group emitted more negative calls than control animals in the light/shock period. (B) Similarly, iuGC group displayed increased percentage of freezing behavior in comparison with control animals. On the test day, animals were exposed six times to cue but no shock was given. iuGC presented an increase in the number of USVs (C) and in freezing time (D). (E) iuGC animals are hyper-reactive throughout time, since they emit more context-induced $22 \mathrm{kHz}$ vocalizations than control group, especially in the first $60 \mathrm{~s}$. Upon first cue exposure (ON period), both groups increased the number of USVs with no major differences between them. However, upon second cue exposure, iuGC animals emitted more negative USVs than controls and remained over-reactive during time. CONT, control animals; iuGC, in utero GC exposed animals; USVs, ultrasonic vocalizations. ${ }^{*} p<0.05$, ${ }^{*} p<0.001,{ }^{* *} p<0.0001, \delta$ : trend, $p=0.130$; (A-E) $n=8-16$. we have performed IHC against ChAT, the key enzyme in ACh synthesis. The number of ChAT-positive cells in the LDT was significantly higher in iuGC animals when compared to control group (Figures 3A,B; 49\% increase; $U=3, p=0.026$ ). The PPT, a LDT adjacent region, also presented substantial increase in the number of ChAT-positive cells (Figures 3C,D; $U=2, p=0.024$ ).
On the contrary, the nucleus basalis of Meynert did not show any significant alteration in the number of cholinergic positive cells (Figures 3E,F; $U=8, p=0.315$ ). Other regions such as the NAc (core and shell), rich in cholinergic interneurons, also did not show any differences in the number of ChAT-positive cells (Figures 3G,H; core: $U=13, p=0.927$; shell: $U=6, p=0.164$ ). 
A

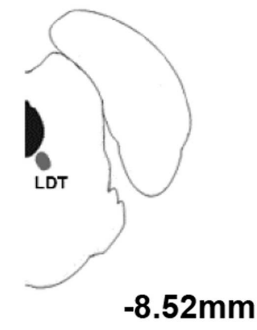

C

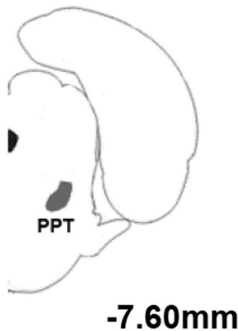

E

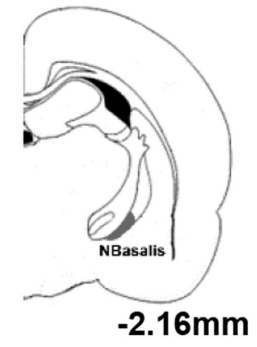

G

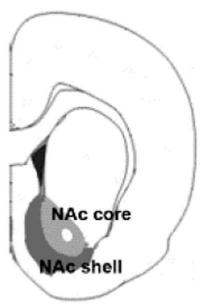

$-1.16 \mathrm{~mm}$
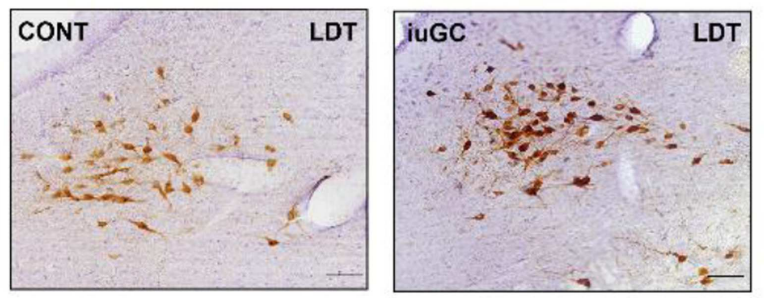

B

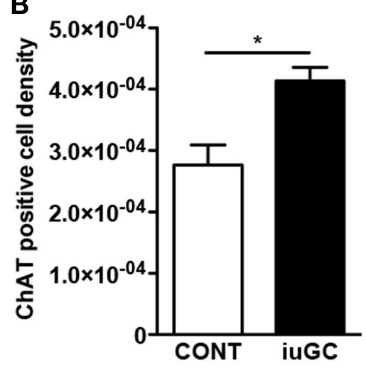

D

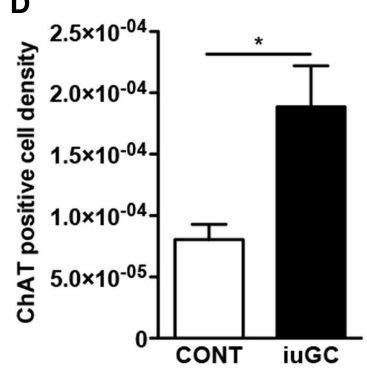

F
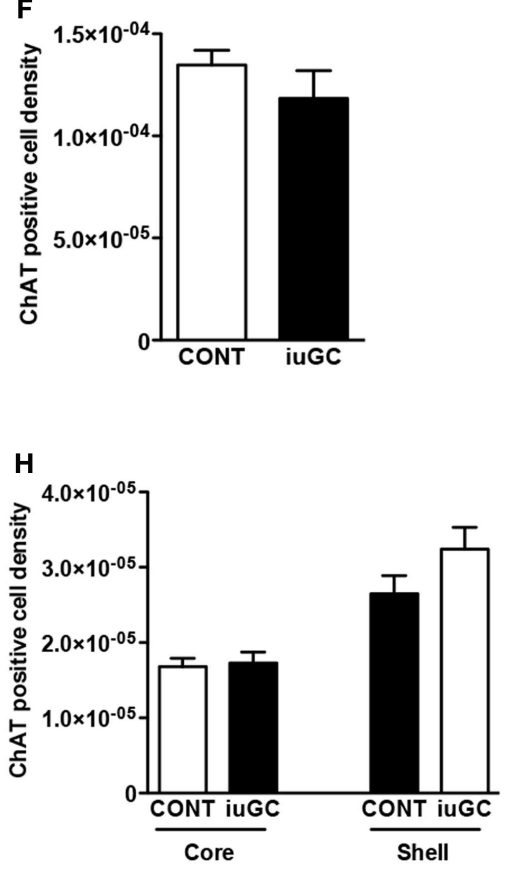

H

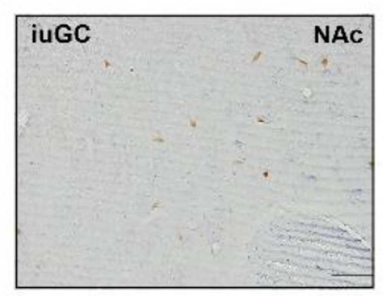

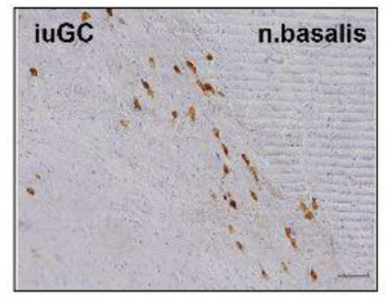

Core
FIGURE 3 | Mesopontine cholinergic de-regulation in iuGC animals. (A) LDT ChAT immunohistochemistry revealed that iuGC animals presented increased ChAT expression (B). (C,D) Similarly, iuGC animals present increased ChAT staining in the PPT region. Conversely, no differences were found in ChAT expression in the nucleus basalis of Meynert (E,F) or in the nucleus accumbens core or shell $\mathbf{( G , H ) . ~ R e p r e s e n t a t i v e ~ i m a g e s ~ o f ~ c o r o n a l ~ b r a i n ~ s e c t i o n s ; ~ n u m b e r s ~}$ represent distance in $\mathrm{mm}$ posterior to bregma. CONT, control animals; iuGC, in utero GC exposed animals; LDT, laterodorsal tegmental nucleus; NAc, nucleus accumbens; PPT, pedunculopontine tegmental nucleus. ${ }^{*} p<0.05,{ }^{* *} p<0.001,{ }^{* *} p<0.0001$; (A) $n=4-7$. Scale bars: $50 \mu \mathrm{m}$.
Because iuGC can induce relevant structural changes, we measured the volume and number of cells in the LDT and PPT nuclei. No statistical differences regarding the volume of LDT were found between control and iuGC animals (Figures 4A-C; volume: $U=5, p=0.486$; cell numbers: $U=3, p=0.200)$. In the PPT, no significant differences in volume and cell numbers were found 

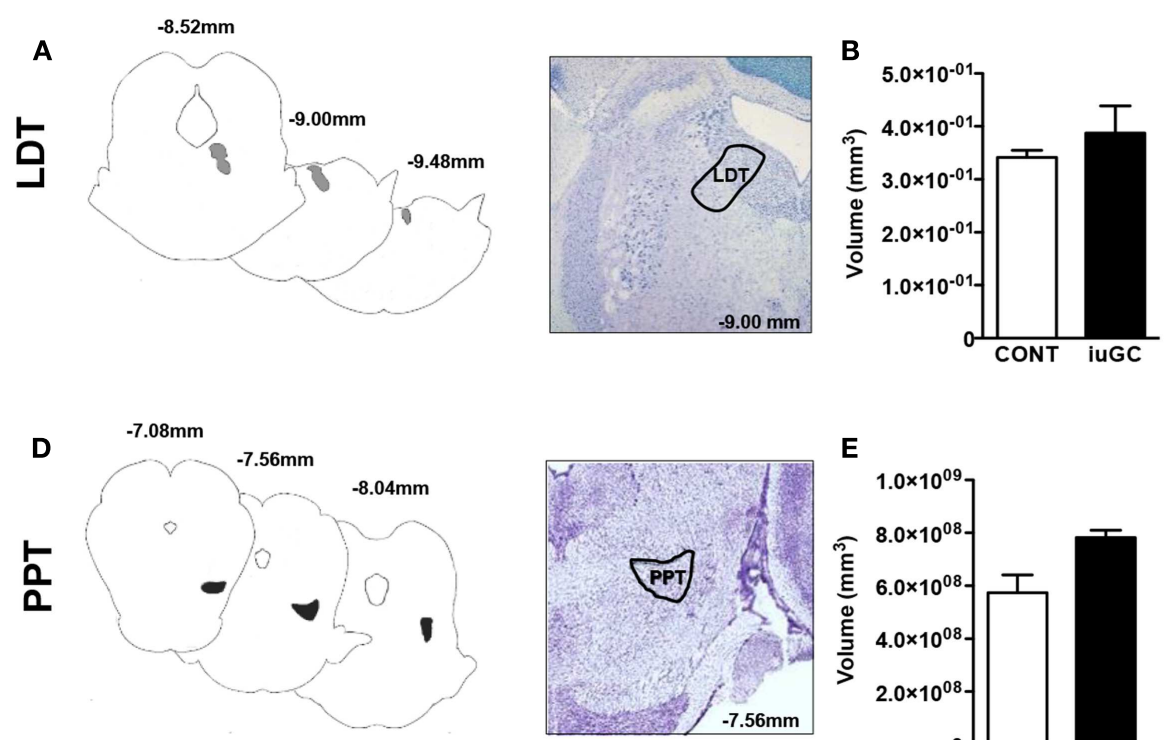

E

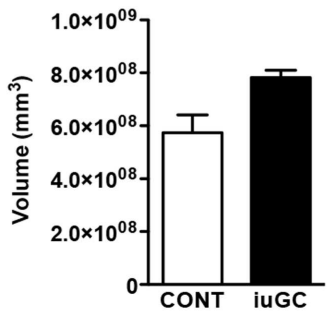

C

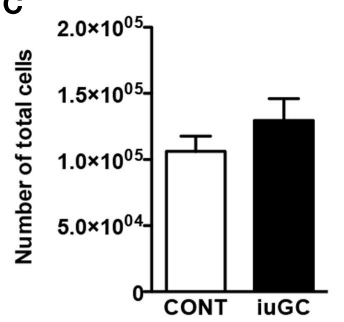

F

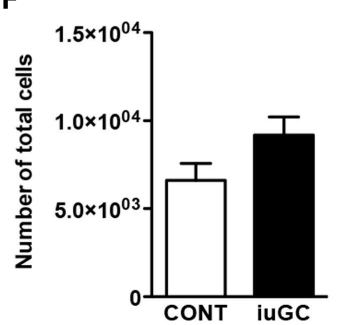

FIGURE 4 | Stereological data of LDT and PPT regions. No major differences were observed in the volumes and cell numbers of LDT (A-C) and PPT regions (D-F) of iuGC animals when compared to controls. Representative images of coronal brain sections; numbers represent distance in millimeters posterior to bregma. CONT, control animals; iuGC, in utero GC exposed animals; LDT, laterodorsal tegmental nucleus; PPT, pedunculopontine tegmental nucleus. (B,C) $n=5-6$; (E,F) $n=4$.
(Figures 4D-F; volume: $U=2, p=0.114$; cell numbers: $U=2$, $p=0.229$ ).

To assess if the observed increase in ChAT-positive cells in iuGC group was translated into augmented ACh release, we measured the levels of $\mathrm{ACh}$ in cholinoceptive regions. A trend for increased ACh levels in the hypothalamus and the amygdala was found (data not shown).

\section{CHOLINERGIC NEURONS IN THE LDT AND PPT ARE DIFFERENTIALLY ACTIVATED UPON ADVERSE STIMULUS IN IUGC ANIMALS}

To better determine the impact of iuGC in cholinergic neurons, and the relevance of such changes in reaction to adverse stimuli, we evaluated neuronal activation patterns using c-fos labeling in combination with ChAT after the fear-conditioning protocol.

Briefly, animals were subjected to the modified fearconditioning protocol, and sacrificed $90 \mathrm{~min}$ after stimuli on the test day. As depicted in Figure 5, after an adverse stimulus, iuGC animals presented a significant increase $(85 \%)$ in the number of c-fos-positive cells in the LDT (Figures 5A,B; $U=0, p=0.008$ ). The number of ChAT-positive cells was also substantially augmented (64\%) in iuGC animals when compared to control animals (Figure 5C; $U=0, p=0.014$ ).

Regarding the PPT, after the adverse stimuli, iuGC animals present 2.5 times more c-fos-positive cells than control animals (Figures 5E,F; $U=0, p=0.002$ ). Similarly, the number of ChATpositive cells was also increased in iuGC animals (Figure 5G; $U=0, p=0.002$ ).

To determine if the observed increase in c-fos-positive cells was due to enhanced cholinergic activation, we also quantified the number of c-fos/ChAT-positive cells after fear conditioning.
Remarkably, iuGC animals presented a substantial increase $(186 \%)$ in the number of c-fos/ChAT-positive cells in the LDT in comparison to control animals (Figure 5D; $U=4, p=0.029$ ). Similarly, in the PPT, we observed that iuGC animals display a four-time increase in the number of double c-fos/ChAT-positive cells (Figure $5 \mathrm{H}$; 3.358 vs. $0.801 \%, U=4.5, p=0.030$ ).

\section{DISCUSSION}

Confirming previous findings $(12,14,48)$, we observed that iuGC animals present an anxious phenotype. In the EPM, iuGC animals presented a decrease in the time and entries in the open arms when compared with control animals, in accordance with the $\mathrm{L} / \mathrm{D}$ test, where they spent less time in the anxiogenic compartment. Moreover, iuGC animals emitted more $22 \mathrm{kHz}$ negative calls and presented enhanced freezing behavior in the confined cage paradigm. These differences were eliminated by habituation. This is in accordance with previous work showing that rats that are highly anxious tend to vocalize more often and present augmented freezing time during aversive stimuli than rats that display low anxiety-like trait (35). In the fear-conditioning paradigm, iuGC animals emitted more negative calls and enhanced freezing behavior on the conditioning day. This suggests an emotional overreactivity of iuGC animals to adverse stimulus. In further support of this idea, iuGC animals emitted more negative calls than control subjects in response to the cue predicting the harmful stimulus and during ISI, suggesting an over-reactive response. Altogether, our data confirms that iuGC exposure leads to anxious behavior and exacerbated response to stressful events in adulthood, a finding also observed in other stress models (49-51). Similarly, a rat line that displays signs of extreme trait anxiety also presents increased stress vulnerability and reactivity (52). 

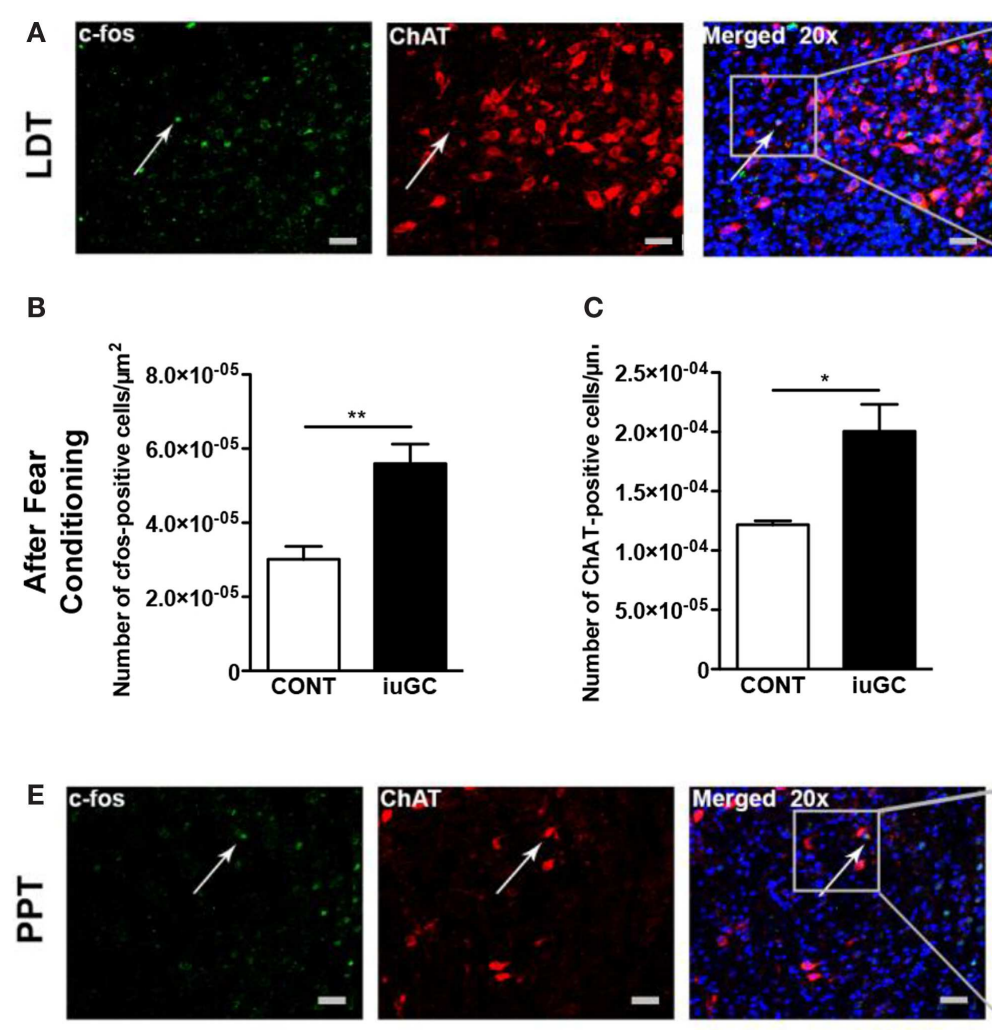

$\mathbf{F}$

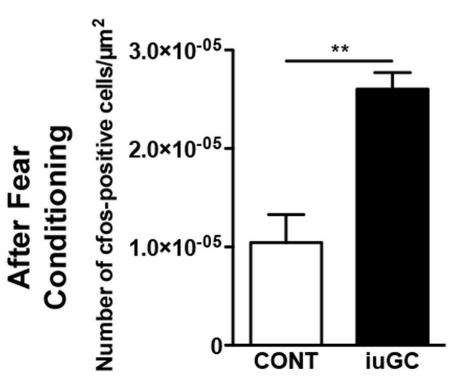

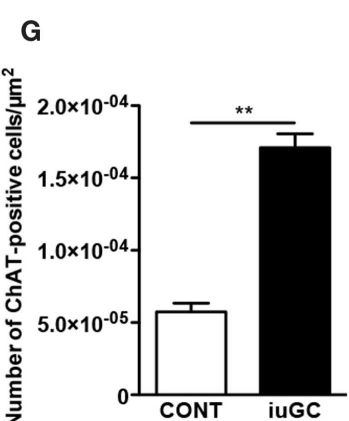
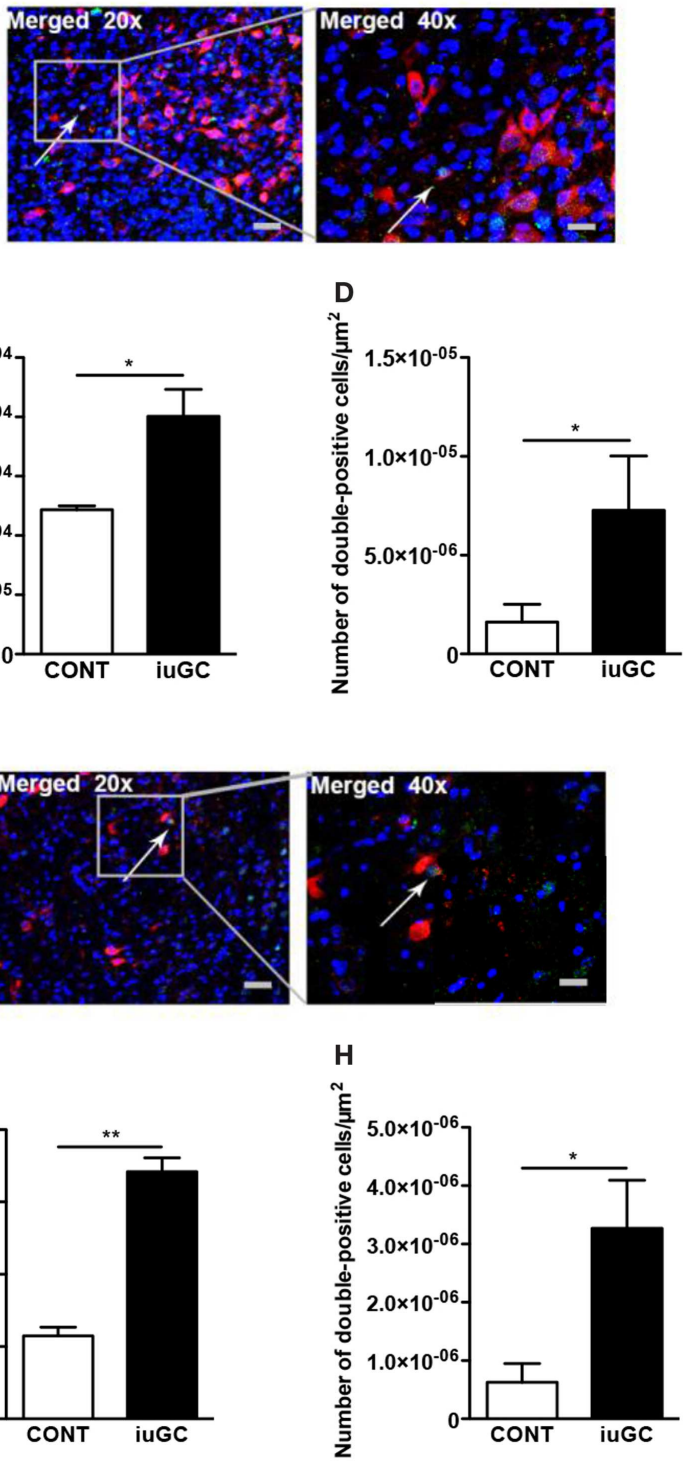

FIGURE 5 | Differential cholinergic activation of the LDT and PPT regions in iuGC animals after an adverse stimulus. (A) Representative images of LDT ChAT (red labeling) and c-fos (green labeling) immunostaining in animals exposed to the fear-conditioning test. (B) Increased c-fos activation in iuGC animals. (C) ChAT expression was increased in iuGC animals in comparison to controls. (D) The number of c-fos/ChAT-positive cells was substantially increased in the LDT of iuGC animals. (E) Representative images of PPT
ChAT and c-fos immunostaining in animals exposed to the fear-conditioning test. (F) Increased c-fos staining in iuGC animals. (G) ChAT expression was augmented in iuGC animals. (H) The number of c-fos/ChAT-positive cells is significantly increased in the PPT of iuGC group. CONT, control animals; iuGC, in utero GC exposed animals; LDT, laterodorsal tegmental nucleus; PPT, pedunculopontine tegmental nucleus. ${ }^{*} p<0.05$, ${ }^{*} p<0.001$; (A-H) $n=6$. Scale bars: $50 \mu \mathrm{m}$.
One remarkable finding was the increased emission of negative calls in iuGC animals, hinting differential activity of the mesopontine cholinergic circuitry, that mainly comprises projections from the LDT region $(43,44,53,54)$. In accordance, we found increased ChAT expression in the LDT of iuGC animals in a basal situation, suggesting overproduction of ACh. Upon exposure to a cue predicting an adverse stimulus, there was a marked increase in the number of c-fos/ChAT-positive cells, potentially explaining iuGC behavioral response. Indeed it was shown that LDT activation induces a complex state of defensiveness, critical for alarm responses to dangerous stimuli $(44,45)$. Our results are in accordance with previous work, in which they have shown that pharmacological stimulation of the LDT lead to an increase in the emission of $22 \mathrm{kHz}$ USVs (44). In addition, the increased LDT cholinergic signaling could also be implicated in the impaired negative HPA axis feedback of iuGC animals (12) since LDTarising ACh enhances ACTH and CRF release $(28,55,56)$. On the other hand, GC also modulate cholinergic signaling (19), suggesting a reciprocal ACh-GC control that may culminate in a vicious loop. 
Alike the LDT, iuGC animals presented increased PPT cholinergic signaling both in a basal situation and after exposure to the cue predicting an adverse stimulus. Apart from the classical role of PPT in sleep and arousal, lesion studies suggest a role in anxiety modulation, although both anxiogenic and anxiolytic effects were found depending on the degree and type of lesion. Excitotoxic and electrolytic PPT lesions induce an anxiogenic-like status $(57,58)$, contrary to one study that suggests a slight anxiolytic effect (59). On the other hand, ibotenic lesions seem to reduce anxious-like phenotype (60). Apart from distinct technical procedures, it has been suggested that opposing results may arise from damage in different sub-regions within the PPT (pars compacta vs. dissipata) or in surrounding regions, namely the cuneiform nucleus $(59,61)$.

This programing effect of iuGC exposure is not so surprising considering the importance of GC receptors for the maturation of medial septal and hippocampal cholinergic neurons (62, 63), yet, more studies need to be performed to understand how GC exert long-lasting functional and molecular changes in these neurons.

In utero glucocorticoid exposure-induced alterations in the mesopontine cholinergic pathway may go beyond a direct effect of cholinergic inputs on behavioral output. For instance, LDTdependent cholinergic activation of VTA evokes dopamine release in the NAc $(20,21,64)$ driving motivational and reward behaviors $(65,66)$. However, these findings are somewhat contradictory to the VTA-accumbal hypodopaminergia and anhedonia observed in iuGC animals $(6,14,15)$. One possible explanation is that the sustained augmented LDT-VTA ACh signaling could desensitize or alter the expression/epigenetic status of the nicotinic/muscarinic receptors as a compensatory mechanism. Indeed, GC or acute stress can induce prominent $\mathrm{ACh}$ release in specific brain regions $(46,67,68)$, and transiently change the expression levels of different cholinergic players through c-fos binding to promoter regions of target genes in order to maintain the homeostasis (19). Additional studies focusing on the regulation of the expression of different cholinergic players by GC will be critical to better comprehend our findings.

In summary, our results show for the first time that prenatal GC exposure programs the mesopontine cholinergic pathway, leading to cholinergic hyperactivation of both the LDT and PPT, which in turn can underlie the anxious behavior and enhanced stress reactivity observed in these animals.

\section{ACKNOWLEDGMENTS}

Authors would like to thanks Tircia Santos and Mariana Cerqueira (3B's group) for the technical help with the fluorimeter. This project was supported by a grant of Institute for the Study of Affective Neuroscience (ISAN) and by Janssen Neuroscience Prize. Sónia Borges, Carina SoaresCunha, Miguel M. Carvalho, Ana P. Ventura-Silva, and Ana João Rodrigues have Fundação para a Ciência e Tecnologia (FCT) fellowships (Sónia Borges: SFRH/BD/89936/2012; Carina Soares-Cunha: SFRH/BD/51992/2012; Miguel M. Carvalho: SRFH/BD/51061/2010, Ana P. Ventura-Silva: SFRH/BD/52078/ 2013; Ana João Rodrigues: SFRH/BPD/33611/2009).

\section{REFERENCES}

1. Heim C, Nemeroff CB. The role of childhood trauma in the neurobiology of mood and anxiety disorders: preclinical and clinical studies. Biol Psychiatry (2001) 49:1023-39. doi:10.1016/S0006-3223(01)01157-X

2. Heim C, Nemeroff CB. Neurobiology of early life stress: clinical studies. Semin Clin Neuropsychiatry (2002) 7:147-59. doi:10.1053/scnp.2002.33127

3. Seckl JR, Meaney MJ. Glucocorticoid programming. Ann N Y Acad Sci (2004) 1032:63-84. doi:10.1196/annals.1314.006

4. Matthews SG. Antenatal glucocorticoids and programming of the developing CNS. Pediatr Res (2000) 47:291-300. doi:10.1203/00006450-200003000-00003

5. McArthur S, McHale E, Dalley JW, Buckingham JC, Gillies GE. Altered mesencephalic dopaminergic populations in adulthood as a consequence of brief perinatal glucocorticoid exposure. J Neuroendocrinol (2005) 17:475-82. doi:10.1111/j.1365-2826.2005.01331.x

6. Leao P, Sousa JC, Oliveira M, Silva R, Almeida OF, Sousa N. Programming effects of antenatal dexamethasone in the developing mesolimbic pathways. Synapse (2007) 61:40-9. doi:10.1002/syn.20341

7. Rodrigues AJ, Leao P, Carvalho M, Almeida OF, Sousa N. Potential programming of dopaminergic circuits by early life stress. Psychopharmacology (Berl) (2011) 214:107-20. doi:10.1007/s00213-010-2085-3

8. Wyrwoll CS, Holmes MC. Prenatal excess glucocorticoid exposure and adult affective disorders: a role for serotonergic and catecholamine pathways. Neuroendocrinology (2012) 95:47-55. doi:10.1159/000331345

9. Vallee M, Mayo W, Dellu F, Le Moal M, Simon H, MacCari S. Prenatal stress induces high anxiety and postnatal handling induces low anxiety in adult offspring: correlation with stress-induced corticosterone secretion. $J$ Neurosci (1997) 17:2626-36.

10. Welberg LA, Seckl JR, Holmes MC. Prenatal glucocorticoid programming of brain corticosteroid receptors and corticotrophin-releasing hormone: possible implications for behaviour. Neuroscience (2001) 104:71-9. doi:10.1016/S03064522(01)00065-3

11. MacCari S, Darnaudery M, Morley-Fletcher S, Zuena AR, Cinque C, Van Reeth O. Prenatal stress and long-term consequences: implications of glucocorticoid hormones. Neurosci Biobehav Rev (2003) 27:119-27. doi:10.1016/S0149-7634(03) 00014-9

12. Oliveira M, Bessa JM, Mesquita A, Tavares H, Carvalho A, Silva R, et al. Induction of a hyperanxious state by antenatal dexamethasone: a case for less detrimental natural corticosteroids. Biol Psychiatry (2006) 59:844-52. doi:10.1016/j.biopsych.2005.08.020

13. Oliveira M, Rodrigues AJ, Leao P, Cardona D, Pego JM, Sousa N. The bed nucleus of stria terminalis and the amygdala as targets of antenatal glucocorticoids: implications for fear and anxiety responses. Psychopharmacology (Berl) (2012) 220:443-53. doi:10.1007/s00213-011-2494-y

14. Rodrigues AJ, Leao P, Pego JM, Cardona D, Carvalho MM, Oliveira M, et al. Mechanisms of initiation and reversal of drug-seeking behavior induced by prenatal exposure to glucocorticoids. Mol Psychiatry (2012) 17:1295-305. doi:10.1038/mp.2011.126

15. Borges S, Coimbra B, Soares-Cunha C, Miguel Pego J, Sousa N, Joao Rodrigues A. Dopaminergic modulation of affective and social deficits induced by prenatal glucocorticoid exposure. Neuropsychopharmacology (2013) 38:2068-79. doi:10.1038/npp.2013.108

16. Henderson Z, Sherriff FE. Distribution of choline acetyltransferase immunoreactive axons and terminals in the rat and ferret brainstem. J Comp Neurol (1991) 314:147-63. doi:10.1002/cne.903140114

17. Oakman SA, Faris PL, Kerr PE, Cozzari C, Hartman BK. Distribution of pontomesencephalic cholinergic neurons projecting to substantia nigra differs significantly from those projecting to ventral tegmental area. J Neurosci (1995) 15:5859-69.

18. Holmstrand EC, Sesack SR. Projections from the rat pedunculopontine and laterodorsal tegmental nuclei to the anterior thalamus and ventral tegmental area arise from largely separate populations of neurons. Brain Struct Funct (2011) 216:331-45. doi:10.1007/s00429-011-0320-2

19. Kaufer D, Friedman A, Seidman S, Soreq H. Acute stress facilitates longlasting changes in cholinergic gene expression. Nature (1998) 393:373-7. doi: $10.1038 / 30741$

20. Blaha CD, Allen LF, Das S, Inglis WL, Latimer MP, Vincent SR, et al. Modulation of dopamine efflux in the nucleus accumbens after cholinergic stimulation of the ventral tegmental area in intact, pedunculopontine tegmental nucleus-lesioned, 
and laterodorsal tegmental nucleus-lesioned rats. J Neurosci (1996) 16: 714-22.

21. Forster GL, Blaha CD. Laterodorsal tegmental stimulation elicits dopamine efflux in the rat nucleus accumbens by activation of acetylcholine and glutamate receptors in the ventral tegmental area. Eur J Neurosci (2000) 12:3596-604. doi:10.1046/j.1460-9568.2000.00250.x

22. Floresco SB, West AR, Ash B, Moore H, Grace AA. Afferent modulation of dopamine neuron firing differentially regulates tonic and phasic dopamine transmission. Nat Neurosci (2003) 6:968-73. doi:10.1038/nn1103

23. Lodge DJ, Grace AA. The laterodorsal tegmentum is essential for burst firing of ventral tegmental area dopamine neurons. Proc Natl Acad Sci U S A (2006) 103:5167-72. doi:10.1073/pnas.0510715103

24. Berse B, Blusztajn JK. Modulation of cholinergic locus expression by glucocorticoids and retinoic acid is cell-type specific. FEBS Lett (1997) 410:175-9. doi:10.1016/S0014-5793(97)00568-1

25. Meshorer E, Erb C, Gazit R, Pavlovsky L, Kaufer D, Friedman A, et al. Alternative splicing and neuritic mRNA translocation under long-term neuronal hypersensitivity. Science (2002) 295:508-12. doi:10.1126/science.1066752

26. Ohmori N, Itoi K, Tozawa F, Sakai Y, Sakai K, Horiba N, et al. Effect of acetylcholine on corticotropin-releasing factor gene expression in the hypothalamic paraventricular nucleus of conscious rats. Endocrinology (1995) 136:4858-63. doi:10.1210/en.136.11.4858

27. Llorente I, Lizcano F, Alvarez R, Diez N, Sopena M, Gil MJ, et al. Cholinergic modulation of spontaneous hypothalamic-pituitary-adrenal activity and its circadian variation in man. J Clin Endocrinol Metab (1996) 81:2902-7. doi:10.1210/jc.81.8.2902

28. Rhodes ME, Rubin RT. Functional sex differences ('sexual diergism') of central nervous system cholinergic systems, vasopressin, and hypothalamic-pituitaryadrenal axis activity in mammals: a selective review. Brain Res Brain Res Rev (1999) 30:135-52. doi:10.1016/S0165-0173(99)00011-9

29. Ross SA, Wong JY, Clifford JJ, Kinsella A, Massalas JS, Horne MK, et al. Phenotypic characterization of an alpha 4 neuronal nicotinic acetylcholine receptor subunit knock-out mouse. J Neurosci (2000) 20:6431-41.

30. Salas R, Pieri F, Fung B, Dani JA, De Biasi M. Altered anxiety-related responses in mutant mice lacking the beta4 subunit of the nicotinic receptor. J Neurosci (2003) 23:6255-63.

31. Booker TK, Butt CM, Wehner JM, Heinemann SF, Collins AC. Decreased anxiety-like behavior in beta3 nicotinic receptor subunit knockout mice. Pharmacol Biochem Behav (2007) 87:146-57. doi:10.1016/j.pbb.2007.04.011

32. National Institutes of Health. The effect of antenatal steroids for fetal maturation on perinatal outcomes. NIH Consens Statement (1994) 12(2):1-24.

33. Miracle X, Di Renzo GC, Stark A, Fanaroff A, Carbonell-Estrany X, Saling E. Guideline for the use of antenatal corticosteroids for fetal maturation. J Perinat Med (2008) 36:191-6. doi:10.1515/JPM.2008.032

34. Smith LM, Qureshi N, Chao CR. Effects of single and multiple courses of antenatal glucocorticoids in preterm newborns less than 30 weeks' gestation. J Matern Fetal Med (2000) 9:131-5. doi:10.1002/(SICI)1520-6661(200003/04)9:2<131: :AID-MFM9>3.3.CO;2-D

35. Borta A, Wohr M, Schwarting RK. Rat ultrasonic vocalization in aversively motivated situations and the role of individual differences in anxiety-related behavior. Behav Brain Res (2006) 166:271-80. doi:10.1016/j.bbr.2005.08.009

36. Keuker JI, Vollmann-Honsdorf GK, Fuchs E. How to use the optical fractionator: an example based on the estimation of neurons in the hippocampal CA1 and CA3 regions of tree shrews. Brain Res Brain Res Protoc (2001) 7:211-21. doi:10.1016/S1385-299X(01)00064-2

37. Paxinos G, Watson C. The Rat Brain in Stereotaxic Coordinates. 6th ed. San Diego: Academic Press (2007).

38. West MJ, Slomianka L, Gundersen HJ. Unbiased stereological estimation of the total number of neurons in the subdivisions of the rat hippocampus using the optical fractionator. Anat Rec (1991) 231:482-97. doi:10.1002/ar.1092310411

39. Gundersen HJ, Jensen EB, Kieu K, Nielsen J. The efficiency of systematic sampling in stereology - reconsidered. J Microsc (1999) 193:199-211. doi:10.1046/ j.1365-2818.1999.00457.x

40. Gundersen HJ, Jensen EB. The efficiency of systematic sampling in stereology and its prediction. J Microsc (1987) 147:229-63. doi:10.1111/j.1365-2818.1987. tb02837.x

41. Ling EA, Paterson JA, Privat A, Mori S, Leblond CP. Investigation of glial cells in semithin sections. I. Identification of glial cells in the brain of young rats. J Comp Neurol (1973) 149:43-71. doi:10.1002/cne.901490104
42. Peinado MA, Quesada A, Pedrosa JA, Martinez M, Esteban FJ, Del Moral ML, et al. Light microscopic quantification of morphological changes during aging in neurons and glia of the rat parietal cortex. Anat Rec (1997) 247:420-5. doi:10.1002/(SICI)1097-0185(199703)247:3<420::AID-AR14>3.3.CO;2-O

43. Brudzynski SM, Barnabi F. Contribution of the ascending cholinergic pathways in the production of ultrasonic vocalization in the rat. Behav Brain Res (1996) 80:145-52. doi:10.1016/0166-4328(96)00029-0

44. Brudzynski SM. Pharmacological and behavioral characteristics of $22 \mathrm{kHz}$ alarm calls in rats. Neurosci Biobehav Rev (2001) 25:611-7. doi:10.1016/S01497634(01)00058-6

45. Brudzynski SM. Ultrasonic calls of rats as indicator variables of negative or positive states: acetylcholine-dopamine interaction and acoustic coding. Behav Brain Res (2007) 182:261-73. doi:10.1016/j.bbr.2007.03.004

46. Imperato A, Puglisi-Allegra S, Casolini P, Angelucci L. Changes in brain dopamine and acetylcholine release during and following stress are independent of the pituitary-adrenocortical axis. Brain Res (1991) 538:111-7. doi:10.1016/0006-8993(91)90384-8

47. Tinsley MR, Quinn JJ, Fanselow MS. The role of muscarinic and nicotinic cholinergic neurotransmission in aversive conditioning: comparing Pavlovian fear conditioning and inhibitory avoidance. Learn Mem (2004) 11:35-42. doi:10.1101/lm.70204

48. Roque S, Oliveira TG, Nobrega C, Barreira-Silva P, Nunes-Alves C, Sousa N, et al. Interplay between depressive-like behavior and the immune system in an animal model of prenatal dexamethasone administration. Front Behav Neurosci (2011) 5:4. doi:10.3389/fnbeh.2011.00004

49. Romeo RD, Mueller A, Sisti HM, Ogawa S, McEwen BS, Brake WG. Anxiety and fear behaviors in adult male and female C57BL/6 mice are modulated by maternal separation. Horm Behav (2003) 43:561-7. doi:10.1016/S0018506X(03)00063-1

50. Tsoory M, Cohen H, Richter-Levin G. Juvenile stress induces a predisposition to either anxiety or depressive-like symptoms following stress in adulthood. Eur Neuropsychopharmacol (2007) 17:245-56. doi:10.1016/j. euroneuro.2006.06.007

51. Uchida S, Hara K, Kobayashi A, Funato H, Hobara T, Otsuki K, et al. Early life stress enhances behavioral vulnerability to stress through the activation of REST4-mediated gene transcription in the medial prefrontal cortex of rodents. J Neurosci (2010) 30:15007-18. doi:10.1523/JNEUROSCI.1436-10.2010

52. Landgraf R, Wigger A. Born to be anxious: neuroendocrine and genetic correlates of trait anxiety in HAB rats. Stress (2003) 6:111-9. doi:10.1080/ 1025389031000104193

53. Bihari A, Hrycyshyn AW, Brudzynski SM. Role of the mesolimbic cholinergic projection to the septum in the production of $22 \mathrm{kHz}$ alarm calls in rats. Brain Res Bull (2003) 60:263-74. doi:10.1016/S0361-9230(03)00041-8

54. Brudzynski SM. Communication of adult rats by ultrasonic vocalization: biological, sociobiological, and neuroscience approaches. ILAR J (2009) 50:43-50. doi:10.1093/ilar.50.1.43

55. Risch SC, Kalin NH, Janowsky DS. Cholinergic challenges in affective illness: behavioral and neuroendocrine correlates. J Clin Psychopharmacol (1981) 1:186-92. doi:10.1097/00004714-198107000-00003

56. Hasey G, Hanin I. The cholinergic-adrenergic hypothesis of depression reexamined using clonidine, metoprolol, and physostigmine in an animal model. Biol Psychiatry (1991) 29:127-38. doi:10.1016/0006-3223(91)90041-J

57. Podhorna J, Franklin KB. Lesions of the pedunculopontine tegmental nucleus increase anxiety in rats. Neuroreport (1998) 9:1783-6. doi:10.1097/00001756199806010-00020

58. Leri F, Franklin KBJ. Learning impairments caused by lesions to the pedunculopontine tegmental nucleus: an artifact of anxiety? Brain Res (1998) 807:187-92. doi:10.1016/S0006-8993(98)00762-8

59. Homs-Ormo S, Coll-Andreu M, Satorra-Marin N, Arevalo-Garcia R, MorgadoBernal I. Effects of pedunculopontine tegmental nucleus lesions on emotional reactivity and locomotion in rats. Brain Res Bull (2003) 59:495-503. doi:10.1016/S0361-9230(02)00966-8

60. Satorra-Marín N, Homs-Ormo S, Arévalo-García R, Morgado-Bernal I, CollAndreu M. Effects of pre-training pedunculopontine tegmental nucleus lesions on delayed matching- and non-matching-to-position in a T-maze in rats. Behav Brain Res (2005) 160:115-24. doi:10.1016/j.bbr.2004.11.022

61. Walker SC, Winn P. An assessment of the contributions of the pedunculopontine tegmental and cuneiform nuclei to anxiety and neophobia. Neuroscience (2007) 150:273-90. doi:10.1016/j.neuroscience.2007.09.018 
62. Takahashi LK, Goh CS. Glucocorticoid facilitation of cholinergic development in the rat hippocampus. Neuroscience (1998) 83:1145-53. doi:10.1016/S03064522(97)00472-7

63. Guijarro C, Rutz S, Rothmaier K, Turiault M, Zhi Q, Naumann T, et al. Maturation and maintenance of cholinergic medial septum neurons require glucocorticoid receptor signaling. J Neurochem (2006) 97:747-58. doi:10.1111/j.14714159.2006.03728.x

64. Miller AD, Blaha CD. Midbrain muscarinic receptor mechanisms underlying regulation of mesoaccumbens and nigrostriatal dopaminergic transmission in the rat. Eur J Neurosci (2005) 21:1837-46. doi:10.1111/j.1460-9568.2005. 04017.x

65. Berridge KC. The debate over dopamine's role in reward: the case for incentive salience. Psychopharmacology (Berl) (2007) 191:391-431. doi:10.1007/s00213006-0578-x

66. Wise RA. Dopamine and reward: the anhedonia hypothesis 30 years on. Neurotox Res (2008) 14:169-83. doi:10.1007/BF03033808

67. Gilad GM, Mahon BD, Finkelstein Y, Koffler B, Gilad VH. Stress-induced activation of the hippocampal cholinergic system and the pituitaryadrenocortical axis. Brain Res (1985) 347:404-8. doi:10.1016/0006-8993(85) 90209-4

68. Mark GP, Rada PV, Shors TJ. Inescapable stress enhances extracellular acetylcholine in the rat hippocampus and prefrontal cortex but not the nucleus accumbens or amygdala. Neuroscience (1996) 74:767-74. doi:10.1016/03064522(96)00211-4

Conflict of Interest Statement: The authors declare that the research was conducted in the absence of any commercial or financial relationships that could be construed as a potential conflict of interest.

Received: 28 October 2013; paper pending published: 16 November 2013; accepted: 25 November 2013; published online: 13 December 2013.

Citation: Borges S, Coimbra B, Soares-Cunha C, Ventura-Silva AP, Pinto L, Carvalho MM, Pêgo J-M, Rodrigues AJ and Sousa N (2013) Glucocorticoid programing of the mesopontine cholinergic system. Front. Endocrinol. 4:190. doi: 10.3389/fendo.2013.00190

This article was submitted to Neuroendocrine Science, a section of the journal Frontiers in Endocrinology.

Copyright (c) 2013 Borges, Coimbra, Soares-Cunha, Ventura-Silva, Pinto, Carvalho, Pêgo, Rodrigues and Sousa. This is an open-access article distributed under the terms of the Creative Commons Attribution License (CC BY). The use, distribution or reproduction in other forums is permitted, provided the original author(s) or licensor are credited and that the original publication in this journal is cited, in accordance with accepted academic practice. No use, distribution or reproduction is permitted which does not comply with these terms. 